\title{
Alfvén-wave transmission and test-particle acceleration in parallel relativistic shocks
}

\author{
R. Vainio ${ }^{1}$, J. J. P. Virtanen ${ }^{2}$, and R. Schlickeiser ${ }^{3}$ \\ 1 Department of Physical Sciences, P O Box 64, 00014 University of Helsinki, Finland \\ 2 Tuorla Observatory ${ }^{\star}$, University of Turku, 21500 Piikkiö, Finland \\ 3 Institut für Theoretische Physik IV, Ruhr-Universität Bochum, 44780 Bochum, Germany
}

Received 2 July 2003 / Accepted 28 July 2003

\begin{abstract}
Alfvén-wave transmission through super-Alfvénic parallel relativistic shock waves is studied. We calculate the wave transmission coefficients for given shock properties. We show (i) that the Alfvén waves downstream the shock wave are propagating predominantly anti-parallel to the flow direction for low-Mach-number shocks, as in the case of non-relativistic shocks; and (ii) that for high-Mach-number ultra-relativistic shocks the forward and backward downstream waves are in equipartition. For low Alfvénic Mach numbers, the scattering center compression ratio of the shock, thus, becomes large and the spectral index of accelerated test particles approaches the limit $\sigma \rightarrow 1$ at shock waves approaching the critical value of the quasi-Newtonian Alfvénic Mach number (i.e., the ratio of upstream fluid and Alfvén proper speeds), which depends on the shock properties, and equals the square root of the compression ratio at the test-wave limit. Although the inclusion of the wave electromagnetic and velocity fields to the shock jump conditions is likely to decrease the scattering-center compression ratio for shocks with critical Mach numbers, values significantly above the gas compression ratio can be expected for such shocks. Particle acceleration in weak relativistic shocks propagating in magnetized astrophysical jets may, therefore, be substantially more efficient than predicted by models neglecting turbulent electric fields.
\end{abstract}

Key words. acceleration of particles - ISM: cosmic rays - galaxies: jets - relativity - shock waves - waves

\section{Introduction}

Explosive astrophysical events, such as supernova remnants, gamma-ray bursts (GRBs) and jets of active galactic nuclei (AGNi), are of immense importance for high-energy astrophysics, both as intense non-thermal radiation emitters as well as probable source sites for accelerating cosmic ray particles. The leading collapsar (Woosley 1993; Paczynski 1998) and supranova (Vietri \& Stella 1998) models for gamma-ray bursts involve relativistic outflows (Piran 1999) that emanate from a compact source, where due to strong stellar envelope magnetic fields and/or explosions in a pre-existing pulsar wind bubble (Kennel \& Coroniti 1984) anisotropic relativistic outflow velocities result with initial Lorentz factors $\gamma_{0} \approx 300$. AGN jets can be regarded as channeled collimated relativistic outflow components $\left(\gamma_{0} \approx 30\right)$ propagating in the dynamic jet medium which might be identical to the ambient interstellar medium (e.g., Dermer \& Schlickeiser 1992; Urry \& Padovani 1995). In both source classes it is of interest to unravel the basic

Send offprint requests to: R. Vainio,

e-mail: rami.vainio@helsinki.fi

* Tuorla Observatory is part of the Väisälä Institute for Space Physics \& Astronomy, University of Turku, Finland. physical processes that convert the huge kinetic outflow energy into observable non-thermal radiation.

Because the signal speeds (speed of sound and Alfvén speed) of the surrounding interstellar and intergalactic plasmas are typically smaller than the relativistic jet speeds, the formation of magnetized relativistic shock waves is a direct consequence of these violent explosive events. According to current understanding, energetic charged particles are efficiently accelerated at shocks by the first-order Fermi mechanism with power law distribution functions in particle rigidity, and subsequently give rise to the observed non-thermal radiation. By simple equipartition arguments, it is anticipated that a considerable fraction of the outflow energy is transformed into these power law distributions of energetic particles. Time-dependent modeling of the non-thermal synchrotron, synchrotron-self-Compton and external Compton cooling of energetic pairs in the evolving outflow source region are then performed to explain multiwavelength spectra and light curves in the optically thin case. Analogous calculations in an optically thick environment very often start from energetic hadrons that via cascades involving inelastic hadron-hadron-interactions and/or photomeson interactions provide copious amounts of secondary pairs.

Simple leptonic cooling models are remarkably successful in explaining the observed multi-wavelength spectra of 
AGN jets (see Dermer \& Schlickeiser 2002 and references therein) and GRB afterglows (e.g., Dermer et al. 2000). The observed rapid variability in the TeV light curves from Mrk 501 and Mrk 421 (Gaidos et al. 1996; Quinn et al. 1999; Aharonian et al. 1999, 2001) is attributed in these models to a variable injection rate into the acceleration process (e.g., Mastichiadis \& Kirk 1997; Kirk \& Mastichiadis 1999), probably resulting from the shock wave propagating through a highly structured, non-uniform medium.

Acceleration of particles in non-relativistic shock waves is governed by the first-order Fermi mechanism: particles get accelerated by converging magnetic scattering centers at the shock. At non-relativistic shocks, the theory is referred to as diffusive shock acceleration (Axford et al. 1977; Krymsky 1977; Bell 1978; Blandford \& Ostriker 1978). In the simplest version of the theory, the predicted power-law spectral index of the energy spectrum of the accelerated particles is given solely by the compression ratio of the shock, $r$, as $\sigma=(r+2) /(r-1)$. The same mechanism relying on converging scattering centers at the shock is also operative at relativistic shock waves (Peacock 1981), although no analytical expression between the compression ratio and the spectral index of the accelerated particles can be found in this case (Kirk \& Schneider 1987). The spectral index of the accelerated particles is, however, mainly determined by the compression ratio even for relativistic shocks although, unlike for non-relativistic shocks, details of particle scattering have also an effect on the spectral index (Kirk \& Duffy 1999; Kirk et al. 2000).

As noted already by Bell (1978), however, the compression ratio felt by the accelerated particles is not necessarily equal to the gas compression ratio. This is because the turbulent magnetic field responsible for the accelerated-particle scattering is generally not static in the local plasma frame. If the turbulent fields consist of low-frequency plasma waves, it is possible to transform their time dependence away by considering particle scattering in the frame co-moving with the waves. Thus, the spectral index of the particles is determined by the scattering-center compression ratio, i.e., the ratio of average shock-frame speeds of the scattering centers upstream and downstream of the shock. Acceleration of relativistic particle populations at parallel non-relativistic shocks taking account of the finite wave speeds relative to the plasma was discussed by Vainio \& Schlickeiser $(1998,1999,2001)$. They noted that it is possible to determine the average wave speeds by calculating the downstream wave amplitudes from the assumed properties of the upstream waves by using the shock jump conditions (McKenzie \& Westphal 1969). Vainio \& Schlickeiser (1998) noted (i) that the effect of finite wave speeds relative to the local rest frame to particle acceleration is very important in fastmode shocks with low Alfvénic Mach numbers and (ii) that test-particle acceleration in such shocks is extremely efficient because the transmission of waves through the (super-Alfvénic) shock yields values of the scattering-center compression ratio much above the gas compression ratio.

So far, studies attempting to calculate the spectral index of accelerated particles at relativistic shocks have completely overlooked the possible role of finite wave speeds in these shocks despite the fact that both theoretical and observational estimates of the magnetic fields and densities in relativistic jets often predict relativistic values of the Alfvén speed (Appl \& Camenzind 1988). In this paper, we take the first attempt to calculate the scattering-center compression ratio of a relativistic shock generalizing our previous results of Alfvén wave transmission and test particle acceleration (Vainio \& Schlickeiser 1998) to the relativistic regime. In this first study, we will restrict the discussion to parallel shock waves. We will also adopt a test-wave approach and neglect the effects of wave electromagnetic and velocity fields to the gas compression ratio of the shock. Self-consistent modeling would have to take these into account at the lowest Alfvénic Mach numbers (Vainio \& Schlickeiser 1999). We will also neglect the effects of possible pressure anisotropies in the upstream region (Vainio \& Schlickeiser 2001), and the possible effects of the particles themselves to the downstream wave fields (Vainio 2001). These effects will, however, be qualitatively discussed in Sect. 4. A brief outline of the used notation is given as an Appendix.

\section{Alfvén-wave transmission}

In this section we will calculate the transmission coefficients of Alfvén waves in relativistic shocks, i.e., the amplitudes of the forward and backward propagating Alfvén waves downstream of the shock from assumed amplitudes of the waves in the upstream region. The approach is based on the assumption that wave lengths are much larger than the shock thickness, which allows one to consider the relativistic MHD jump conditions in their usual form despite the fact that the wave fields are actually slowly temporally variable and inhomogeneous on large scales.

\subsection{Wave transmission coefficients}

We consider Alfvén waves ahead and behind a super-Alfvénic shock front. Appl \& Camenzind (1988) noted that the magnetic fields upstream and downstream of the shock are co-planar. By using the relativistic jump conditions, they calculated the ratio of shock-frame tangential magnetic fields upstream (subscript 1) and downstream (subscript 2) as (Eqs. (24) and (25) of Appl \& Camenzind 1988)

$q \equiv \frac{B_{2 \mathrm{t}}}{B_{1 \mathrm{t}}}=r \frac{\mu_{2} \gamma_{2} M^{2}-\mu_{1} \gamma_{1}}{\mu_{2} \gamma_{2} M^{2}-r \mu_{1} \gamma_{1}}$,

where $M^{2}=u_{1}^{2} / u_{\mathrm{A}}^{2}$ is the quasi-Newtonian Alfvénic Mach number of the flow, $\mu=(\rho+P) / n, n, \rho$, and $P$ are the specific enthalpy, the number density, the total energy density, and the gas pressure (all measured in the local plasma frame), $u$ and $\gamma=\sqrt{1+u^{2}}$ are the proper speed and the Lorentz factor of the flow, $r=\gamma_{2} n_{2} /\left(\gamma_{1} n_{1}\right)$ is the shock-frame compression ratio of the shock, and $u_{\mathrm{A}}$ is the proper Alfvén speed (in units of $c$ ) given by

$u_{\mathrm{A}}^{2}=\frac{B_{0}^{2}}{4 \pi \mu n}$.

Here, $B_{0}$ is the magnitude of the static background magnetic field measured in the plasma frame. We will restrict the discussion to parallel shock waves and to Alfvén waves propagating along the mean magnetic field. This implies that the 
phase speed of the Alfvén waves relative to the plasma is $\beta_{\mathrm{A}}=u_{\mathrm{A}} / \sqrt{1+u_{\mathrm{A}}^{2}}$.

Since we are considering parallel shock waves (i.e., with the ordered magnetic field along the shock normal), the tangential fields are composed of the fluctuating wave fields. We denote the wave-frame magnetic field amplitudes by $\tilde{B}_{ \pm}$, where the subscripts + and - refer to waves propagating parallel or anti-parallel to the flow. The phase speeds of the waves in the shock frame are

$V_{ \pm}=\frac{\beta \pm \beta_{\mathrm{A}}}{1 \pm \beta \beta_{\mathrm{A}}}$,

where $\beta=u / \gamma$. In the wave frame, the wave electric field vanishes, which allows one to give the wave magnetic fields in the shock frame as

$B_{ \pm}=\Gamma_{ \pm} \tilde{B}_{ \pm}$

where $\Gamma_{ \pm}=1 / \sqrt{1-V_{ \pm}^{2}}$. (Note that the magnetic amplitude of the wave in the plasma frame is $\gamma_{\mathrm{A}} \tilde{B}_{ \pm}$with $\gamma_{\mathrm{A}}=\sqrt{1+u_{\mathrm{A}}^{2}}$.) Thus, the tangential fields in the shock frame are obtained as superpositions of the wave amplitudes as

$\boldsymbol{B}_{\mathrm{t}}=\boldsymbol{B}_{+}+\boldsymbol{B}_{-}=\Gamma_{+} \tilde{\boldsymbol{B}}_{+}+\Gamma_{-} \tilde{\boldsymbol{B}}_{-}$.

We consider wave transmission at the test-wave limit and, therefore, calculate how parallel and anti-parallel propagating waves are transmitted across the shock separately. Thus, we assume that $B_{1 \mathrm{t}}=\Gamma_{1 \pm} \tilde{B}_{1 \pm}$ in the upstream region. In the downstream region, however, the magnetic field can not consist of a single wave field only, because this would not be consistent with the continuity of the transverse electric field, $\boldsymbol{n} \times \boldsymbol{E}$, across the shock with $\boldsymbol{n} \| \boldsymbol{V}_{ \pm}$denoting a unit vector along the shock normal. The tangential electric fields on both sides of the shock can be given as

$\boldsymbol{E}=-\Gamma_{+} \boldsymbol{V}_{+} \times \tilde{\boldsymbol{B}}_{+}-\Gamma_{-} \boldsymbol{V}_{-} \times \tilde{\boldsymbol{B}}_{-}$

giving

$\boldsymbol{n} \times \boldsymbol{E}=V_{+} \boldsymbol{B}_{+}+V_{-} \boldsymbol{B}_{-}$,

which, in general, is not consistent with Eqs. (1) and (5) assuming a single wave field on both sides of the shock. Thus, even if the upstream waves are unidirectional, there are always the two wave modes present in the downstream region. It is also readily clear from the equations that since the magnetic fields are co-planar and $\boldsymbol{n} \times \boldsymbol{E}$ is conserved, the two wave magnetic fields in the downstream region are parallel to each other (immediately behind the shock wave). Thus, the equations governing the downstream wave fields can be given simply as

$q B_{1 \pm}=B_{2+}+B_{2-}$

$V_{1 \pm} B_{1 \pm}=V_{2+} B_{2+}+V_{2-} B_{2-}$

and solved for the shock-frame wave transmission coefficients as

$T_{ \pm} \equiv \frac{B_{2 \pm}}{B_{1 \pm}}=\frac{V_{1 \pm}-V_{2 \mp} q}{V_{2 \pm}-V_{2 \mp}}$

$R_{ \pm} \equiv \frac{B_{2 \mp}}{B_{1 \pm}}=\frac{V_{1 \pm}-V_{2 \pm} q}{V_{2 \mp}-V_{2 \pm}}$.
At the non-relativistic limit, $\gamma_{1}, \gamma_{\mathrm{A} 1} \rightarrow 1$, these transmission coefficients reduce to the results of Vainio \& Schlickeiser (1998),

$$
\begin{aligned}
& T_{ \pm} \rightarrow \frac{\sqrt{r}(\sqrt{r}+1)}{2} \frac{M \pm 1}{M \pm \sqrt{r}} \\
& R_{ \pm} \rightarrow \frac{\sqrt{r}(\sqrt{r}-1)}{2} \frac{M \pm 1}{M \mp \sqrt{r}}
\end{aligned}
$$

In our further analysis, it turns out to be useful to define the transmission coefficients also as the ratios of wave-frame amplitudes of the waves. For these, we obtain

$\tilde{T}_{ \pm} \equiv \frac{\tilde{B}_{2 \pm}}{\tilde{B}_{1 \pm}}=\frac{\Gamma_{1 \pm}}{\Gamma_{2 \pm}} \frac{V_{1 \pm}-V_{2 \mp} q}{V_{2 \pm}-V_{2 \mp}}$
$\tilde{R}_{ \pm} \equiv \frac{\tilde{B}_{2 \mp}}{\tilde{B}_{1 \pm}}=\frac{\Gamma_{1 \pm}}{\Gamma_{2 \mp}} \frac{V_{1 \pm}-V_{2 \pm} q}{V_{2 \mp}-V_{2 \pm}}$.

\subsection{Small-amplitude Alfvén waves}

Assuming the Alfvén waves to have so small amplitudes that they do not affect the shock dynamics we can considerably simplify the analysis. In this case, the jump conditions that determine the shock dynamics reduce to those of a hydrodynamical shock. Thus, the energy equation is

$\gamma_{1} u_{1} \mu_{1} n_{1}=\gamma_{2} u_{2} \mu_{2} n_{2}$

which, by using the mass conservation law $u_{1} n_{1}=u_{2} n_{2}$, gives

$\mu_{1} \gamma_{1}=\mu_{2} \gamma_{2}$.

Thus, Eq. (1) reduces to

$q=r \frac{M^{2}-1}{M^{2}-r}$.

For the flow to remain super-Alfvénic everywhere, we have to require $M>M_{\mathrm{c}} \equiv \sqrt{r}$, where $M_{\mathrm{c}}$ is a critical value of the Mach number below which a hydrodynamical shock becomes non-evolutionary (e.g., Kirk \& Duffy 1999).

In addition to the wave amplitudes, we need equations for the wave numbers, as well. As argued by Vainio \& Schlickeiser (1998), waves conserve their shock-frame frequencies when crossing the shock front. Thus,

$\tilde{k}_{2+}=\frac{\Gamma_{1 \pm} V_{1 \pm}}{\Gamma_{2+} V_{2+}} \tilde{k}_{1 \pm} ; \quad \tilde{k}_{2-}=\frac{\Gamma_{1 \pm} V_{1 \pm}}{\Gamma_{2-} V_{2-}} \tilde{k}_{1 \pm}$,

giving the downstream wave-frame wave number of the forward $\left(\tilde{k}_{2+}\right)$ and backward $\left(\tilde{k}_{2-}\right)$ waves as a function of upstream wave-frame wave number $\left(\tilde{k}_{1 \pm}\right)$ and wave phase speeds. (Note that the plasma-frame wave number is given by $\gamma_{\mathrm{A}} \tilde{k}$.) Again, as in the non-relativistic case, the wave number of the backward downstream waves becomes very large as $V_{2-} \rightarrow 0$ with $M \rightarrow M_{\mathrm{c}}$. Because the compression of the wave number is scale independent, the functional form of the wave spectrum is preserved during the transmission process: a power-law spectrum of the upstream waves, for example, generates power-law spectra also for the downstream waves. 
Let us, next, calculate the transmission coefficients at constant wave number $\tilde{k}$ for power-law spectra of upstream and downstream waves, i.e.,

$\tilde{I}_{j}^{ \pm}(\tilde{k})=\mathcal{U}\left(\tilde{k}-\tilde{k}_{0 j}^{ \pm}\right) \tilde{I}_{0 j}^{ \pm}\left(\tilde{k}_{0 j}^{ \pm} / \tilde{k}\right)^{s}$

with $j=1$ [2] for upstream [downstream] waves and $\mathcal{U}(k)$ denoting the step function. These are related to the intensities of the wave magnetic field as

$\tilde{B}_{j \pm}^{2}=\int_{\tilde{k}_{0 j}^{ \pm}}^{\infty} \mathrm{d} \tilde{k} \tilde{I}_{j}^{ \pm}(\tilde{k})=\tilde{I}_{0 j}^{ \pm} \tilde{k}_{0 j}^{ \pm} /(s-1)$.

The transmission coefficients for the wave-frame magnetic field-intensities at constant wave number, thus, become

$\tilde{T}_{\tilde{k} \pm}^{2} \equiv \frac{\tilde{I}_{2}^{ \pm}(\tilde{k})}{\tilde{I}_{1}^{ \pm}(\tilde{k})}=\left(\frac{\Gamma_{1 \pm} V_{1 \pm}}{\Gamma_{2 \pm} V_{2 \pm}}\right)^{s-1} \tilde{T}_{ \pm}^{2}$
$\tilde{R}_{\tilde{k} \pm}^{2} \equiv \frac{\tilde{I}_{2}^{\mp}(\tilde{k})}{\tilde{I}_{1}^{ \pm}(\tilde{k})}=\left(\frac{\Gamma_{1 \pm} V_{1 \pm}}{\Gamma_{2 \mp} V_{2 \mp}}\right)^{s-1} \tilde{R}_{ \pm}^{2}$.

\subsection{The gas compression ratio in the hydrodynamic limit}

We consider shock waves propagating parallel to the mean magnetic field, and neglect the dynamical effects of the wave magnetic field. We restrict the discussion to strong shocks, i.e., those with negligible upstream pressure. In this case, the gas compression ratio $r$ is a solution of (Appl \& Camenzind 1988)

$$
\begin{aligned}
& \gamma_{1}^{2}(\hat{\gamma}-1)^{2} r^{3}-\gamma_{1}^{2}\left(\hat{\gamma}^{2}-1\right) r^{2}+ \\
& \quad\left(\gamma_{1}^{2}-1\right)(2 \hat{\gamma}-1) r-\left(\gamma_{1}^{2}-1\right)=0 .
\end{aligned}
$$

Here, the polytropic index of the downstream gas, $\hat{\gamma}$, is a function of the dimensionless downstream temperature, $\Theta=$ $k_{\mathrm{B}} T_{2} / m c^{2}$. Assuming a thermal equilibrium $\hat{\gamma}$ can be given using the modified Bessel functions of the second kind, $K_{v}(z)$, as (Synge 1957)

$\hat{\gamma}=1+\left[\frac{1}{\Theta}\left(\frac{K_{1}\left(\Theta^{-1}\right)}{K_{2}\left(\Theta^{-1}\right)}-1\right)+3\right]^{-1}$.

The downstream temperature is related to the compression ratio as

$\Theta=\gamma_{1} \gamma_{2} \beta_{1}^{2} \frac{r-1}{r^{2}}$

in case of a hydrodynamic shock (Appl \& Camenzind 1988).

We solve the compression ratio iteratively using the following scheme: (i) fix the value of the polytropic index to $\hat{\gamma}=\frac{4}{3}$, if $\gamma_{1}>2$, and to $\hat{\gamma}=\frac{5}{3}$, if $\gamma_{1}<2$; (ii) solve the compression ratio $r$ numerically from Eq. (24) noting that the physical root is in the range $1 /(\hat{\gamma}-1)<r<(\hat{\gamma}+1) /(\hat{\gamma}-1)$; (iii) calculate a new value for $\hat{\gamma}$ by using $\beta_{2}=\beta_{1} / r$ and Eqs. (25) and (26); (iv) repeat the steps (ii)-(iii) until $\hat{\gamma}$ differs from the value obtained in the previous round by less than a predefined convergence criterion. The gas compression ratio and the downstream polytropic index as a function of the proper speed of the shock are given in Fig. 1.

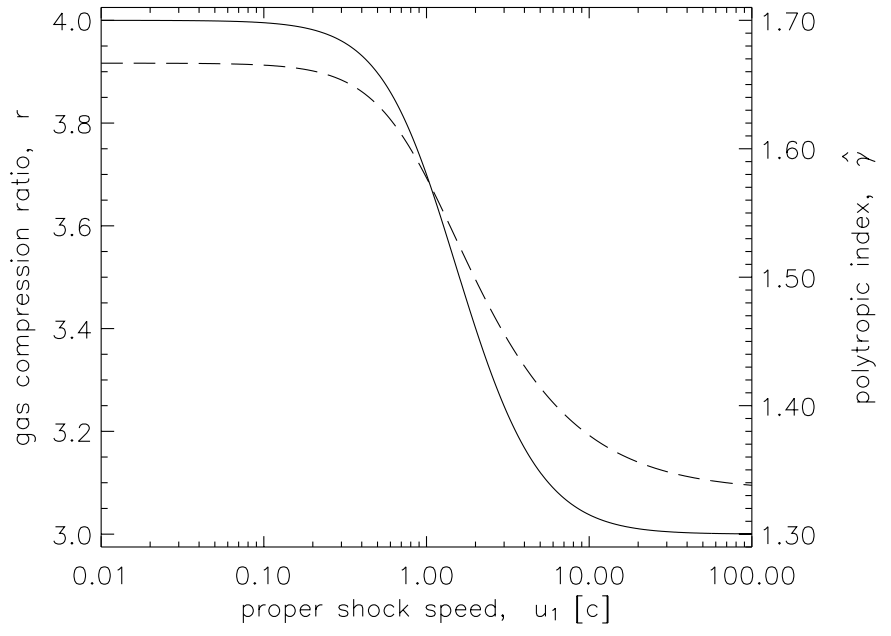

Fig. 1. The gas compression ratio $r$ (solid curve) and the polytropic in$\operatorname{dex} \hat{\gamma}$ (dashed curve) of the downstream gas in a parallel shock propagating into a cold medium.

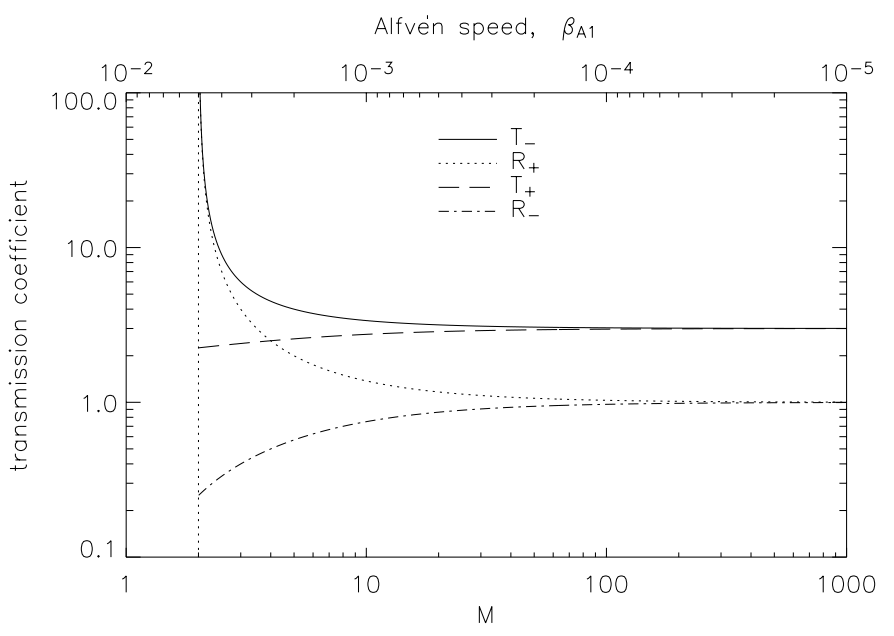

Fig. 2. Alfvén-wave transmission coefficients in a parallel nonrelativistic shock $\left(\beta_{1}=0.01, \hat{\gamma}=\frac{5}{3}\right.$, and $\left.r=4\right)$ propagating into a cold medium. Note that the results as a function of Mach number apply to parallel shocks with any non-relativistic value of $\beta_{1}$.

\subsection{Wave transmission at non-relativistic shocks}

Let us first study the wave transmission coefficients as a function of Mach number by fixing the value of the shock speed to a non-relativistic value and varying the Alfvén speed. We have computed the wave transmission coefficients as a function Alfvénic Mach number for a non-relativistic $\left(\beta_{1}=0.01\right)$ parallel shock propagating in a cold plasma with a polytropic index of $\hat{\gamma}=\frac{5}{3}$ and a compression ratio of $r=4$ (Fig. 2). Note that as long as the shock speed is non-relativistic, the same transmission coefficients as a function of Mach number apply, but the axis for the Alfvén speed, of course, applies only for the chosen value of $\beta_{1}$.

At low Mach numbers, $M^{2} \sim r$, the waves propagate predominantly backward relative to the flow direction, no matter what the state of the upstream wave field is. The physical reason for this is that a finite value of an electric field component for the backward waves, required to satisfy the jump 


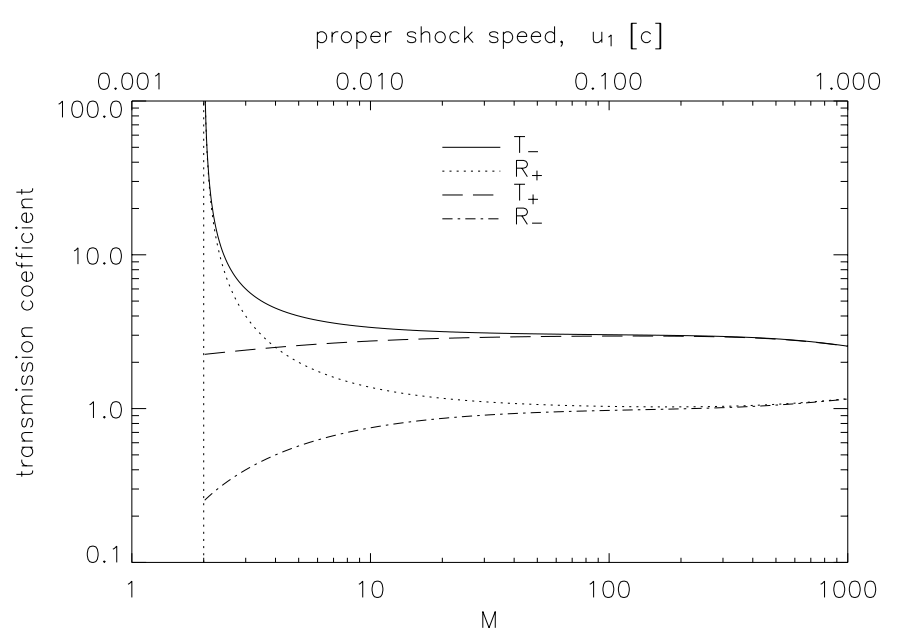

Fig. 3. Alfvén-wave transmission coefficients, as measured in the shock frame, in a parallel shock propagating into a medium with proper Alfvén speed $u_{\mathrm{A}}=0.001 \mathrm{c}$.

conditions, requires very large values of the magnetic field amplitude, if $V_{2-} \rightarrow 0+$, i.e., $M^{2} \rightarrow r+$. The infinite values of the transmission coefficients occur because of the assumption of the negligible wave pressure and Poynting flux. When these are taken into account, the compression ratio of the shock becomes smaller staying below $r<M^{2}$ (Vainio \& Schlickeiser 1999) and, thus, the infinite values of $T_{-}$and $R_{+}$are avoided. At high Mach numbers, the transmission coefficients approach the values $T_{ \pm} \rightarrow 3$ and $R_{ \pm} \rightarrow 1$.

\subsection{Wave transmission for fixed $u_{\mathrm{A}}$ and variable $\gamma_{1}$}

Let us, next, study the wave transmission coefficients as a function of Mach number by fixing the value of the Alfvén speed and varying the shock speed. We study the cases of upstream proper Alfvén speed ranging from $u_{\mathrm{A} 1}=0.001 c$ to $10 c$ (Figs. 3-7). The cases with non-relativistic Alfvén speeds $\left(u_{\mathrm{A} 1} \leq 0.1 c\right)$ differ very little from the above results for non-relativistic shocks up to proper shock speeds of about $u_{1} \sim 0.4 c$, but at larger shock speeds relativistic effects lowering the compression ratio of the shock become important. On the other hand, all transmission coefficients are very similar in the cases $u_{\mathrm{A} 1}=1.0 \mathrm{c}$ and $10.0 \mathrm{c}$ indicating that, once the Alfvén speed becomes relativistic, the only relevant parameter in the wave transmission problem is the Mach number, like in the non-relativistic case. Note, however, that (since $r \rightarrow 3$ and $\left.\beta_{\mathrm{A} 2} \rightarrow 0\right)$ at the limits of $M, u_{1} \rightarrow \infty$ the asymptotic values of all the transmission coefficients are $T_{ \pm}, R_{ \pm} \rightarrow \frac{3}{2}$ and, thus, different from the values at non-relativistic shocks. Therefore, in relativistic high-Mach-number shocks the wave fields in the downstream region are close to equipartition, no matter what the propagation direction of the upstream waves might be. At low Mach numbers, the results are qualitatively similar to non-relativistic shocks (Vainio \& Schlickeiser 1998): the downstream wave field is predominantly backward, regardless of the upstream wave propagation direction. The region of backward-wave dominance, however, extends to slightly larger Mach numbers than in non-relativistic shocks.

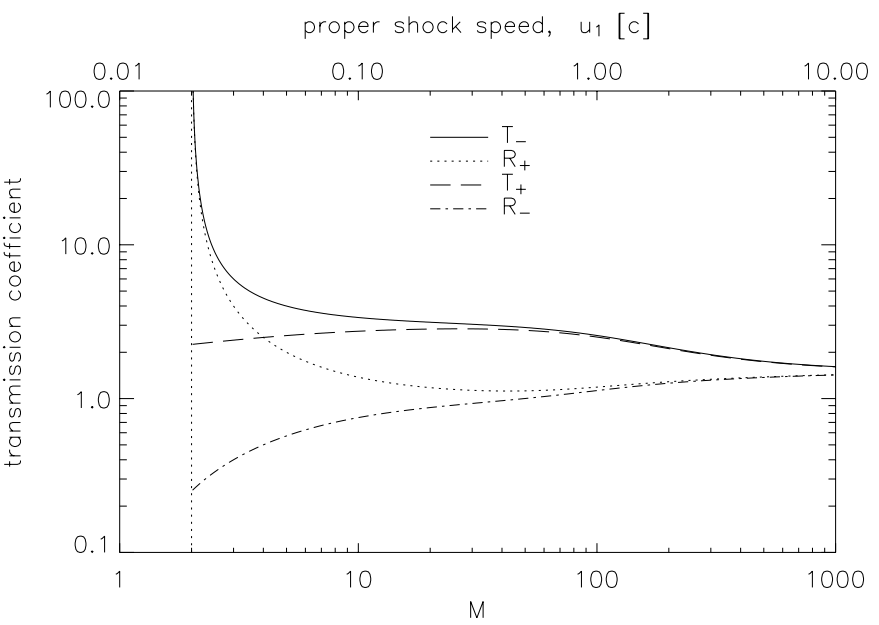

Fig. 4. Alfvén-wave transmission coefficients, as measured in the shock frame, in a parallel shock propagating into a medium with proper Alfvén speed $u_{\mathrm{A}}=0.01 c$.

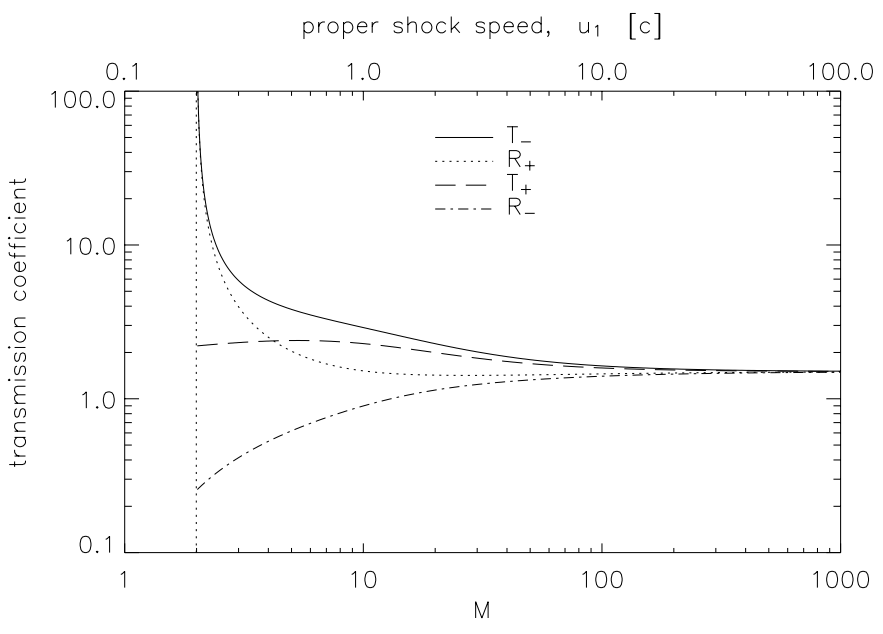

Fig. 5. Alfvén-wave transmission coefficients, as measured in the shock frame, in a parallel shock propagating into a medium with proper Alfvén speed $u_{\mathrm{A}}=0.1 \mathrm{c}$.

\section{Particle acceleration}

Particle acceleration at relativistic shocks is basically due to the same first (and second) order Fermi mechanism as in non-relativistic shocks. The analysis of particle acceleration in relativistic shocks is, however, more complicated than in non-relativistic shocks because the particle distribution is anisotropic. Thus, numerical methods are needed to solve for the spectral index of the accelerated particles in these conditions. Two main classes of numerical methods have been employed so far: (i) Monte Carlo simulations (Ellison et al. 1990; Bednarz \& Ostrowski 1996, 1998) and (ii) solutions based on eigenfunction expansions (Kirk \& Schneider 1987; Kirk et al. 2000). The numerical methods reveal that the spectral index of the accelerated particles is no longer independent of the underlying scattering process: large-angle scattering and pitch-angle diffusion lead to different results concerning this parameter. If pitch-angle diffusion is employed, large upstream Lorentz factors (producing $r \rightarrow 3$ ) seem to yield spectral indices close to the value of $\sigma=2.2$ (in far downstream energy 


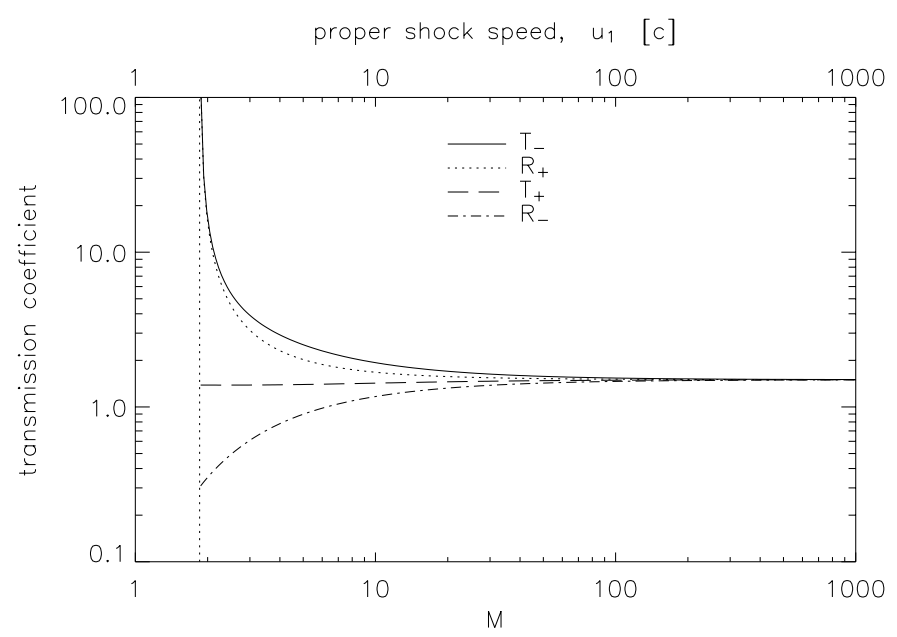

Fig. 6. Alfvén-wave transmission coefficients, as measured in the shock frame, in a parallel shock propagating into a medium with proper Alfvén speed $u_{\mathrm{A}}=1.0 \mathrm{c}$.

spectrum, $p^{2} f(p) \propto p^{-\sigma}$ ) (Bednarz \& Ostrowski 1998; Kirk et al. 2000). All of the studies so far are, however, based on the assumption of frozen-in scattering centers, i.e., they assume that particles scatter elastically in the local rest frame of the plasma. With non-negligible Alfvén speeds, this can no longer be assumed, but one instead has to consider scatterings that are elastic in the frame of the scattering wave (where the wavemagnetic field is static).

We consider an upstream plasma with wave spectra $\tilde{I}_{1}^{+}(\tilde{k})=$ $\tilde{I}_{1}^{-}(\tilde{k})=\mathcal{U}\left(\tilde{k}-\tilde{k}_{01}\right) \tilde{I}_{01}\left(\tilde{k}_{01} / \tilde{k}\right)^{s}$, i.e., a situation where the turbulently evolving forward and backward waves have attained equal power-law intensities in the ambient plasma. Thus, upstream of the shock wave the normalized cross helicity,

$H_{\mathrm{c}}(\tilde{k}) \equiv \frac{\tilde{I}^{+}(\tilde{k})-\tilde{I}^{-}(\tilde{k})}{\tilde{I}^{+}(\tilde{k})+\tilde{I}^{-}(\tilde{k})}$,

at all wave numbers $\tilde{k}>\tilde{k}_{01}$ is $H_{\mathrm{c} 1}=0$. The downstream cross helicity at wave numbers $\tilde{k}>\left(\Gamma_{1+} V_{1+} / \Gamma_{2-} V_{2-}\right) \tilde{k}_{01}$ is given by

$H_{\mathrm{c} 2}=\frac{\tilde{T}_{\tilde{k}+}^{2}+\tilde{R}_{\tilde{k}-}^{2}-\left(\tilde{T}_{\tilde{k}-}^{2}+\tilde{R}_{\tilde{k}+}^{2}\right)}{\tilde{T}_{\tilde{k}+}^{2}+\tilde{R}_{\tilde{k}+}^{2}+\tilde{T}_{\tilde{k}-}^{2}+\tilde{R}_{\tilde{k}-}^{2}}$.

The downstream cross helicities as functions of Mach number (scaled with $M_{\mathrm{c}}$ ) for shocks with $u_{1}=0.1,1,10 \mathrm{c}$ and $s=1.5$ are shown in Fig. 8.

Because the downstream Alfvén speed is at most mildly relativistic, we take the average plasma-frame speed of the downstream scattering centers to be $H_{\mathrm{c} 2} \beta_{\mathrm{A} 2}$. This gives the shock-frame scattering center speed in the downstream region as $V_{k 2}=\left(\beta_{2}+H_{\mathrm{c} 2} \beta_{\mathrm{A} 2}\right) /\left(1+H_{\mathrm{c} 2} \beta_{\mathrm{A} 2} \beta_{2}\right)$. We can now use this value to compute the scattering-center compression ratio of the shock (for $H_{\mathrm{c} 1}=0$ ) as

$r_{k}=\frac{\beta_{1}\left(1+H_{\mathrm{c} 2} \beta_{\mathrm{A} 2} \beta_{2}\right)}{\beta_{2}+H_{\mathrm{c} 2} \beta_{\mathrm{A} 2}}$.

This is presented as a function of Mach number for $s=1.5$ and the three values of $u_{1}$ considered above for $H_{\mathrm{c} 2}$ in Fig. 9. Note that by scaling the Mach number with $M_{\mathrm{c}}=\sqrt{r}$ and the

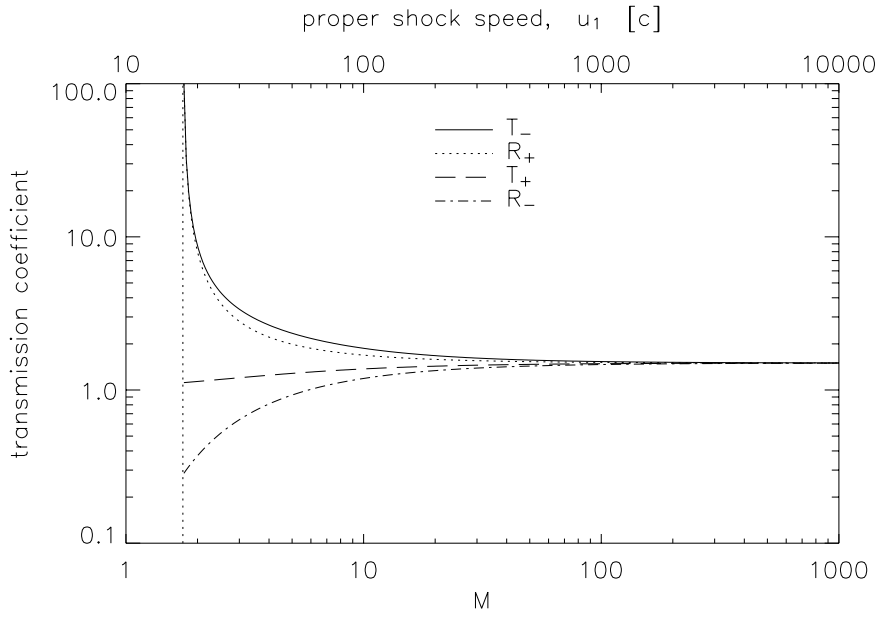

Fig. 7. Alfvén-wave transmission coefficients, as measured in the shock frame, in a parallel shock propagating into a medium with proper Alfvén speed $u_{\mathrm{A}}=10.0 \mathrm{c}$.

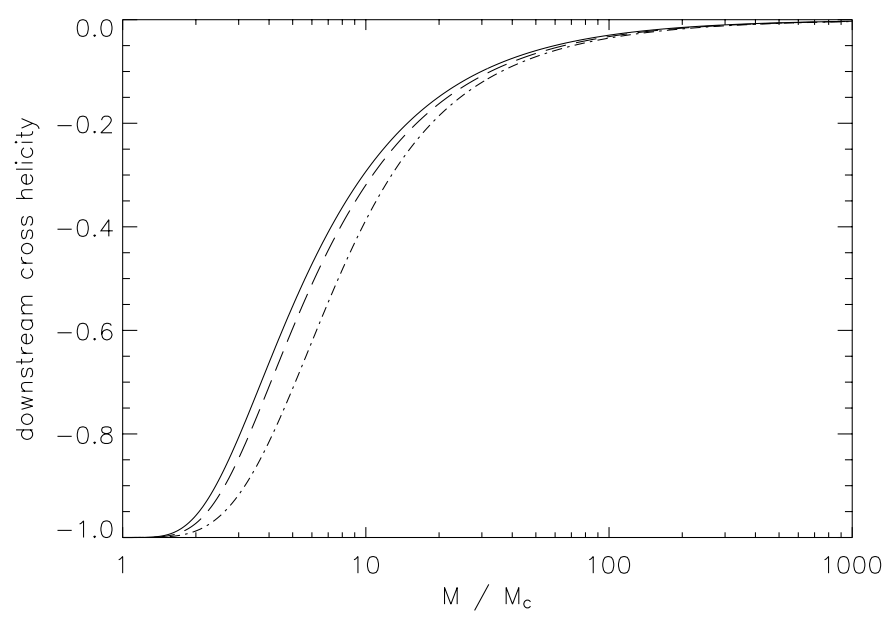

Fig. 8. Cross helicity of the Alfvén waves at constant wave number downstream a parallel shock wave for $u_{1}=0.1 c$ (solid curve, $r \approx$ 3.995), $u_{1}=1.0 c$ (dashed curve, $r \approx 3.700$ ), and $u_{1}=10.0 c$ (dotdashed curve, $r \approx 3.038$ ). The upstream cross helicity is taken to be zero, the spectral index of the waves is $s=1.5$, and $M_{\mathrm{c}}=\sqrt{r}$.

scattering-center compression ratio with $r$, the curves are practically on top of each other.

Two important results can be immediately deduced from the behavior of downstream cross helicity and scattering-center compression ratio at low Mach numbers, i.e., just above the critical one: firstly, accelerated particles feel a very large scattering-center compression ratio at the shock. At the limit of $r_{k}$ increasing toward infinity, particle escape from the shock to far downstream becomes increasingly more difficult, and the spectral index approaches the value of $\sigma \rightarrow 1$, as long as the momentum gain of the particle per each crossing cycle of the shock is a fixed (i.e., momentum independent) fraction of particle momentum. This is the case for ultra-relativistic particles regardless of the shock speed. Secondly, for such low-Machnumber relativistic shocks we can also neglect stochastic acceleration, since the downstream waves are propagating in one direction, only. 


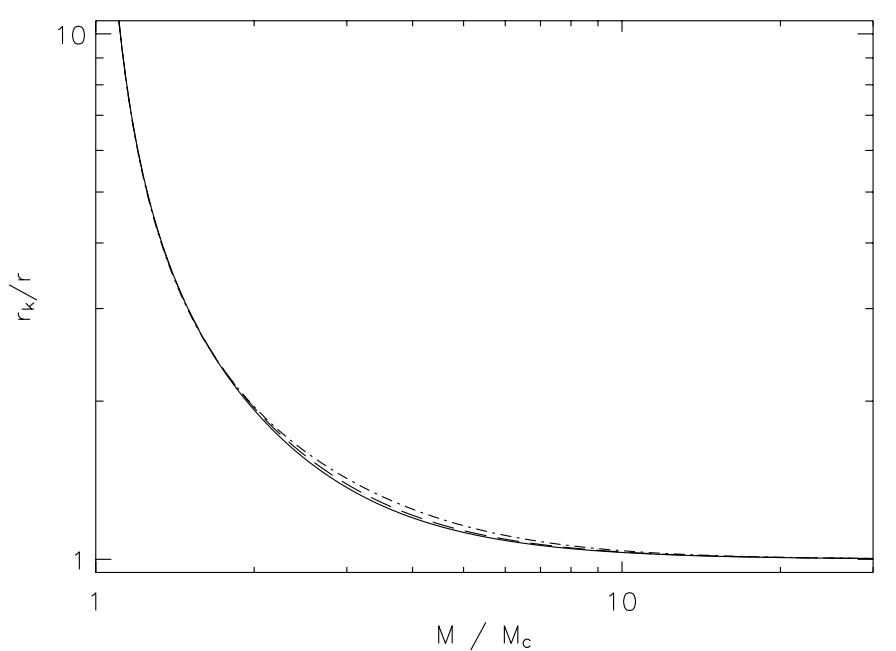

Fig. 9. Scattering center compression ratio, $r_{k}$, of a parallel shock wave for $u_{1}=0.1 c$ (solid curve, $r \approx 3.995$ ), $u_{1}=1.0 c$ (dashed curve, $r \approx$ 3.700), and $u_{1}=10.0 c$ (dot-dashed curve, $r \approx 3.038$ ). The upstream cross helicity is taken to be zero, the spectral index of the waves is $s=1.5$, and $M_{\mathrm{c}}=\sqrt{r}$.

To illustrate the effect of the Alfvén-wave transmission to particle acceleration more quantitatively, we have used Monte Carlo simulations to compute the spectrum of accelerated particles. Our code is a direct generalization to the relativistic regime of the code we have used for studying diffusive shock acceleration at non-relativistic shocks (Vainio et al. 2000). A detailed description of the code will be given elsewhere (Virtanen \& Vainio, in preparation). For simplicity, we employ small-angle scatterings in pitch angle relative to the mean field (i.e., shock normal), $\arccos \mu$, with an isotropic pitch-angle diffusion coefficient $D_{\mu \mu} \propto 1-\mu^{2}$. The validity of this simplification may be questionable in the downstream region, where a form like $D_{\mu \mu} \propto\left(1-\mu^{2}\right) /\left(\mu^{2}+\epsilon^{2}\right)^{1 / 2}$ with $\epsilon \ll 1$ might better describe the scattering conditions in magnetized shocks (Kirk et al. 2000), but the effect of the functional form of $D_{\mu \mu}$ to the accelerated particle spectrum is typically smaller than that of the compression ratio. We employ a single, position dependent scattering center speed that has a value of $\beta_{1}$ upstream and $\beta_{1} / r_{k}$ downstream of the shock wave. Thus, the effect of stochastic acceleration (which we estimated to be small) is not considered in this study. The value of the scatteringcenter compression ratio was varied from $r_{k}=r$ to $8 r$ (see Fig. 9 for the corresponding Mach numbers) and three values of the proper shock speed, $u_{1}=0.1 c, 1.0 c$, and $10.0 c$, were considered. Statistical errors of the fitted spectral indices are of the order of 0.065 or less in all cases. As shown in Fig. 10, the simulated points are close to each other in all cases, by chance, when the scattering-center compression ratios are scaled by the gas compression ratio. As expected, the non-relativistic diffusive shock acceleration theory does not describe the spectral index correctly, if the shock is relativistic. The values, however, seem to converge toward $\sigma \rightarrow 1$ as $r_{k} \rightarrow \infty$, as expected, regardless of the value of $u_{1}$.

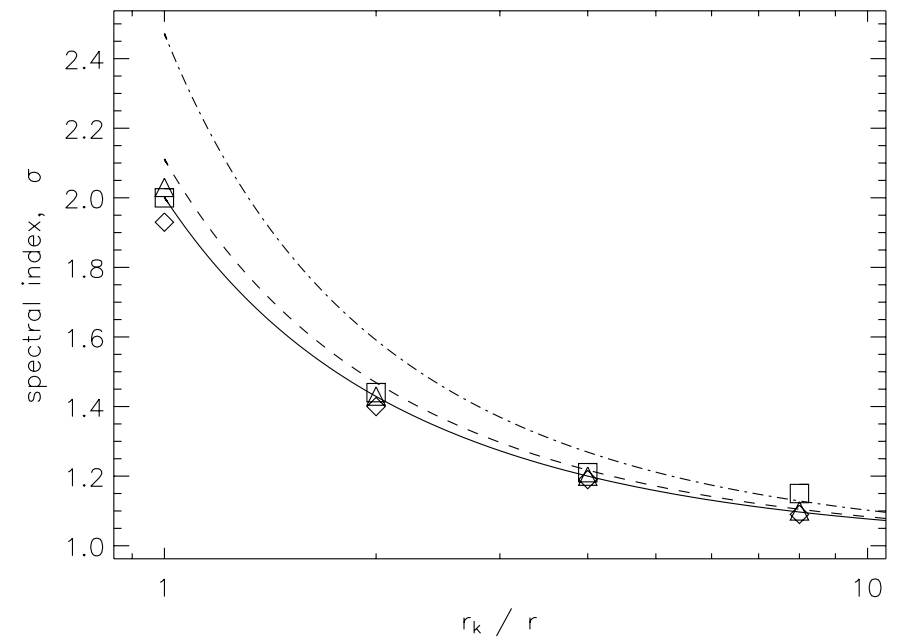

Fig. 10. Spectral index of particles accelerated in a relativistic parallel shock as a function of the scattering-center compression ratio $r_{k}$ for $u_{1}=0.1 c$ (triangles, $r \approx 3.995$ ), $u_{1}=1.0 c$ (squares, $r \approx 3.700$ ), and $u_{1}=10.0 c$ (diamonds, $r \approx 3.038$ ). The solid, dashed, and dot-dashed curves give the results of $\sigma=\left(r_{k}+2\right) /\left(r_{k}-1\right)$ for the three proper speeds, respectively.

\section{Discussion and conclusions}

We have considered Alfvén-wave transmission and test-particle acceleration in parallel relativistic shock waves propagating to a cold plasma. Test-wave approximation has been used, i.e., the electromagnetic and velocity fields of the waves have not been taken into account when determining the gas compression ratio of the shock. Under these admittedly restricting assumptions, it has been shown that the Alfvén waves downstream of a barely super-Alfvénic shock wave are predominantly propagating backward relative to the direction of the flow. This has been shown to strongly affect the scattering-center compression ratio, $r_{k}$, of low-Mach-number shocks, increasing this parameter well above the gas compression ratio, $r$, of the shock. Thus, the computed power-law energy spectrum of particles accelerated in such shock wave is significantly harder than predicted by theories relying on particle scattering off frozen-in magnetic fluctuations. At the test-wave limit, the scattering-center compression ratio becomes infinite and the spectral index of the accelerated particles approaches $\sigma \rightarrow 1$ as the quasi-Newtonian Alfvénic Mach number of the shock, $M=u_{1} \sqrt{4 \pi \mu_{1} n_{1}} / B_{0}$, approaches the critical Mach number $M_{\mathrm{c}}=\sqrt{r}$ from above.

Let us discuss the limitations of our assumptions to the validity of our results. Since we are interested in relativistic shock waves, the most severe limitation comes from our assumption of parallel shock geometry. This is because as the upstream Lorentz factor increases to values $\gamma_{1} \gg 1$, the transverse magnetic fields in the shock frame become amplified, and it is increasingly less probable that the orientation of the shock is such that the ordered transverse fields can be neglected. This should be taken into account in any attempt to apply our results to shocks with $\gamma_{1} \gg 1$. Significant obliqueness of the shock would lower the value of Alfvén-wave speed relative to the shock normal, but at the same time it would lower the gas compression ratio of a low-Mach-number shock wave 
(Appl \& Camenzind 1988). The two effects work in opposite directions in the ratio $r_{k} / r$. Thus, it is not easy to deduce the net effect of the obliqueness to the scattering-center compression ratio; we expect it to get smaller with increasing obliquity, but a quantitative estimate of its effect would require a calculation beyond the scope of the present study.

Another crucial assumption we made was the validity of test-wave approximation. This was the origin of the (unphysical) singularities of the transmission coefficients $T_{-}$and $R_{+}$ at $M \rightarrow \sqrt{r}+$ that lead to the extremely high values of $r_{k}$. The singularity is removed by taking proper account of the transverse wave fields when computing the shock's gas compression ratio. This keeps the value of $r$ in the physical range for fast-mode shocks, $r<M^{2}$, as was demonstrated for nonrelativistic shocks by Vainio \& Schlickeiser (1999). These authors also showed that although the singularities are avoided, the gas compression ratio at shocks with $1<M \lesssim 2$ is only slightly below $M^{2}$ and, therefore, $R_{+}, T_{-}$and $r_{k}$ are still very large and the accelerated particle spectrum significantly harder than the one obtained by using the much smaller $r$ as the value of $r_{k}$. Since the values of the transmission coefficients calculated for the relativistic shocks do not differ too much from the values in non-relativistic shocks at low Mach numbers (see Figs. 2-7), we expect this result to carry over to relativistic shocks as well.

We have also neglected the back-reaction of the accelerated particles to the scattering Alfvén waves in our study. For a relativistic shock this is a valid assumption in the upstream region, because no extended foreshock region with backward streaming accelerated particles is expected if $u_{1}$ is close to the speed of light (Kirk et al. 2000). However, as was demonstrated by Vainio (2001) using numerical simulations, the wave fields downstream the shock wave may become modified by the forward-streaming accelerated particles there. This softens the spectrum somewhat from the hardest test-particle spectra, because the particle streaming away from the shock is likely to damp the backward mode more heavily than the forward one. According to Vainio (2001), the downstream forward wave is actually amplified in non-relativistic shocks, but this might not be the case in relativistic shocks, if the Alfvén speed in the downstream region is a sizable fraction of the particle speed, as in the low-Mach-number shocks considered in this paper.

As demonstrated by Vainio \& Schlickeiser (2001), pressure anisotropies upstream the shock wave can be important for Alfvén wave transmission in non-relativistic shock waves. In relativistic shocks, however, such effects require relativistic thermal speeds in the upstream region, and thus they might not be so important because of the presumably rapid cooling of such plasmas.

As demonstrated above, magneto-static (i.e., frozen-in) turbulence description is still a valid approximation for particle scattering if the Mach number of the shock wave is large. Thus, it is not surprising that ultra-relativistic shocks yielding $\sigma=2.2$ at the magneto-static limit seem to be in good agreement with spectral indices of gamma-ray burst afterglows (Bednarz \& Ostrowski 1998). Not all relativistic shocks are, however, strong shocks. Like in non-relativistic plasmas such as the solar corona, freely propagating (i.e., not driven by pistons) shock waves in highly magnetized plasmas are more likely rather weak with Mach numbers of the order of a few or less. Thus, when applying the theory of relativistic shock acceleration to the astrophysical observations, simplifying assumptions such as the magneto-static limit should be used only after due justification.

In view of our results and the above discussion, we conclude (i) that Alfvén-wave transmission in parallel nonrelativistic shocks leads to downstream wave fields that are propagating backward relative to the flow direction if the quasiNewtonian Alfvénic Mach number of the shock is close to $M=\sqrt{r}$; (ii) that the finite wave speeds relative to the plasma rest frame need to be taken into account when modeling particle acceleration in low-Mach-number relativistic shock waves; (iii) that when these effects are taken into account, (acceleratedparticle) spectral indices as flat as $\sigma \rightarrow 1$ can be accounted for. Self-consistent treatment of Alfvén-wave transmission, as well as modeling of wave transmission in oblique shocks would be important and natural paths for future work on this subject.

\section{Appendix A: Notation used in this work}

This Appendix, provided for the convenience of the reader, explains the most important symbols used in this work. All symbols are also defined in the text, where they are first used.

We use the symbols $\beta, \gamma$, and $u=\gamma \beta$ for bulk speeds, bulk Lorentz factors, and bulk proper speeds of the plasma flow relative to the shock, and $n, P$, and $\rho$ as rest-frame density, pressure and energy density of the plasma. Both the specific enthalpy of the gas and the pitch-angle cosine of an accelerated particle are denoted by $\mu$; the local meaning of the symbol should be fully clear from the context. The Alfvén speed, its Lorentz factor, and the corresponding proper speed, as measured in the local rest frame, are denoted by $\beta_{\mathrm{A}}, \gamma_{\mathrm{A}}$, and $u_{\mathrm{A}}$. As usual, quantities measured upstream [downstream] the shock wave are denoted with subscripts 1 [2]. $M=u_{1} / u_{\mathrm{A} 1}, r=\beta_{1} / \beta_{2}, \hat{\gamma}$ and $\Theta$ denote the quasi-Newtonian Alfvénic Mach-number of the shock, the gas compression ratio, and the polytropic index and the dimensionless temperature of the downstream gas, respectively. All speeds are measured in units of $c$.

The symbols $V$ and $\Gamma$ denote speeds and corresponding Lorentz factors related to Alfvén waves as measured in the shock frame: $V_{ \pm}$and $\Gamma_{ \pm}$are the shock-frame phase speeds and Lorentz factors of the Alfvén waves propagating parallel (+) and anti-parallel (-) to the flow, and $V_{k}$ denotes the scatteringcenter speed, i.e., an averaged wave speed relative to the shock as felt by the accelerated particles. Wave magnetic fields are denoted by $B_{ \pm}$, wave number by $k$, wave spectra by $I^{ \pm}(k)$, and the normalized cross helicity of the waves by $H_{\mathrm{c}}(k)$. Wavetransmission and reflection coefficients are given by $T_{ \pm}$and $R_{ \pm}$, respectively, when measured for the (wave-number integrated) wave amplitudes (see Eqs. (10) and (11)); when measured at constant (wave-frame) wave-number (see Eqs. (22) and (23)), they are further subscripted by $k$. A tilde over all these quantities (e.g., $\tilde{k}_{+}$) indicates that values measured in the appropriate wave frame are considered; symbols without tilde are measured in the shock frame. $r_{k}=V_{k 1} / V_{k 2}, s$, and $\sigma$ denote the 
scattering-center compression ratio of the shock, and the wave and the accelerated-particle spectral indices, respectively.

Acknowledgements. This study is a part of the project "Collective Processes in Astrophysical Plasmas: Waves, Heating and Accelerated Particles" co-funded by the Academy of Finland and Deutscher Akademischer Austauschdienst (DAAD). J.J.P.V. thanks the Finnish Cultural Foundation for financial support. R.S. acknowledges partial support by the Deutsche Forschungsgemeinschaft through Sonderforschungsbereich 591 and by the Bundesministerium für Bildung und Forschung (BMBF) through Verbundforschungsprogramm 05 CH1PCA 6.

\section{References}

Aharonian, F. A., Akhperjanian, A. G., Andronache, M., et al. 1999, A\&A, 350, 757

Aharonian, F., Akhperjanian, A., Barrio, J., et al. 2001, ApJ, 546, 898 Appl, S., \& Camenzind, M. 1988, A\&A, 206, 258

Axford, W. I., Leer, E., \& Skadron, G. 1977, Proc. 15th Internat. Cosmic-Ray Conf. (Plovdiv), 11, 132

Bednarz, J., \& Ostrowski, M. 1996, MNRAS, 283, 447

Bednarz, J., \& Ostrowski, M. 1998, Phys. Rev. Lett., 80, 3911

Bell, A. R. 1978, MNRAS, 182, 147

Blandford, R. D., \& Ostriker, J. P. 1978, ApJ, 221, L29

Dermer, C. D., \& Schlickeiser, R. 1992, Science, 257, 1642

Dermer, C. D., \& Schlickeiser, R. 2002, ApJ, 575, 667
Dermer, C. D., Böttcher, M., \& Chiang, J. 2000, ApJ, 537, 255

Ellison, D. C., Reynolds, S. P., \& Jones, F. C. 1990, ApJ, 360, 702

Gaidos, J. A., Akerlof, C. W., Biller, S.D., et al. 1996, Nature, 383, 319

Kennel, C. F., \& Coroniti, F. V. 1984, ApJ, 283, 694

Kirk, J. G., \& Schneider, P. 1987, ApJ, 315, 425

Kirk, J. G., \& Duffy, P. 1999, J. Phys. G, 25, R163

Kirk, J. G., \& Mastichiadis, A. 1999, Astropart. Phys., 11, 45

Kirk, J. G., Guthmann, A. W., Gallant, Y. A., \& Achterberg, A. 2000, ApJ, 542, 235

Krymsky, G. F. 1977, Dokl. Akad. Nauk. SSSR, 243, 1306

Mastichiadis, A., \& Kirk, J. G. 1997, A\&A, 320, 19

McKenzie, J. F., \& Westphal, K. O. 1969, Planet. Space Sci., 17, 1029

Paczynski, B. 1998, ApJ, 494, L45

Peacock, J. A. 1981, MNRAS, 196, 135

Piran, T. 1999, Phys. Rep., 314, 575

Quinn, J., Bond, I. H., Boyle, P. J., et al. 1999, ApJ, 518, 693

Synge, J. L. 1957, The Relativistic Gas (Amsterdam: North Holland) Urry, C. M., \& Padovani, P. 1995, PASP, 107, 803

Vainio, R. 2001, Proc. 27th Internat. Cosmic-Ray Conf. (Hamburg), 6, 2054

Vainio, R., \& Schlickeiser, R. 1998, A\&A, 331, 793

Vainio, R., \& Schlickeiser, R. 1999, A\&A, 343, 303

Vainio, R., \& Schlickeiser, R. 2001, A\&A, 378, 309

Vainio, R., Kocharov, L., \& Laitinen, T. 2000, ApJ, 528, 1015

Vietri, M., \& Stella, L. 1998, ApJ, 507, L45

Woosley, S. 1993, ApJ, 405, 273 